\title{
ПЕРВИЧНЫЙ ГИПЕРПАРАТИРЕОЗ В МОСКОВСКОЙ ОБЛАСТИ: ДАННЫЕ ВСЕРОССИЙСКОГО РЕГИСТРА НА НАЧАЛО 2020 ГОДА
}

\author{
Крюкова И.В., Тевосян Л.Х., Древаль А.В., Полякова Е.Ю.
}

ГБУЗ МО «Московский областной научно-исследовательский клинический институт им. М.Ф. Владимирского», Москва

ЦЕЛЬ: оценить результаты ведения регистра первичного гиперпаратиреоза (ПГПТ) в Московской области в рамках всероссийской базы данных онлайн-версии Государственного регистра больных с ПГПТ на начало 2020 г.

МАТЕРИАЛЫ И МЕТОДЫ: проведен анализ регистра ПГПТ Московской области (МО) в рамках онлайн-версии всероссийского Государственного регистра больных ПГПТ, который в МО заполняется с момента его разработки - с конца 2016 г. Критерием включения больных в регистр является лабораторно подтвержденный ПГПТ: при уровне ПТГ, превышающем верхнюю границу референсных лабораторных значений, и повышенном или верхненормальном уровне кальция сыворотки крови, зафиксированном дважды. Критерии исключения: вторичный и третичный гиперпаратиреоз, наследственная гипокальциурическая гиперкальциемия и гиперкальциемия, не связанная с повышением ПТГ. Данные регистра в МО оценивались на конец февраля 2020 г.

РЕЗУЛЬТАТЫ: всего в регистр МО на момент исследования были внесены данные 618 больных ПГПТ: 588 женщин и 30 мужчин. Средний возраст пациентов составлял 64,9 года (62,7 года у мужчин и 65,0 года у женщин), средний возраст на момент дебюта заболевания - 61,7 года (у мужчин - 59,4 года, у женщин - 61,8 года). Первые симптомы заболевания у больных выявлялись в среднем в возрасте 58,7 года (в 56,2 года у мужчин и в 58,9 года у женщин).

По данным регистра, выявляемость ПГПТ в МО в 2010 г. составляла 2 больных и к 2014 г. несколько повысилась - до 19 случаев в год. В 2015 г. отмечается значительный рост выявляемости новых случаев заболевания - 46 человек (все больные женщины), в 2016 и в 2017 гг. - по 127 случаев (9 мужчин и 118 женщин в 2016 г., 6 мужчин и 121 женщина — в 2017 г.), в 2018 г. - 117 случаев (5 мужчин и 112 женщин) и в 2019 г. - 139 человек (6 мужчин и 124 женщины).

ПГПТ в активной фазе на момент оценки был у 259 (41,91\%) больных (14 (46,67\%) мужчин и 245 (41,67\%) женщин), ремиссия подтверждена у 335 (54,21\%) пациентов (14 (46,67\%) мужчин и 321 (54,59\%) женщин), рецидив заболевания отмечался у 24 (3,88\%) человек (2 (6,6\%) мужчин и 22 (3,74\%) женщин).

Частота характерных клинических проявлений ПГПТ у больных составила: патология желудочнокишечного тракта у 75,1\% больных (464/618), патология почек - 65,4\% (404/618), остеопороз - 53,7\% (332/618), низкотравматические переломы - у 26,5\% (164/618). Также у больных ПГПТ часто встречались заболевания сердечно-сосудистой системы - 65,9\% (407/618) и артериальная гипертензия - в 59,9\% (370/618).

Средние значения основных лабораторных параметров в зависимости от фазы заболевания составляли:у больных в активной фазе паратиреоидный гормон (ПТГ) - 198 пг/мл, общий кальций - 2,71 ммоль/л, в ремиссии: ПТГ - 57,5 пг/мл, общий кальций крови - 2,34 ммоль/л.

ВЫводы: ведение регистра больных ПГПТ позволяет оценить демографические данные больных, динамику выявляемости новых случаев заболевания, лабораторные показатели, в том числе в зависимости от фазы заболевания, частоту различных осложнений, а также случаи рецидива. В MO, по данным регистра ПГПТ, в последние годы отмечались стабильно высокие показатели выявляемости новых случаев заболевания и высокая частота всех основных клинических появлений заболевания. 